\title{
ИССЛЕДОВАНИЕ ИЗДЕРЖЕК УПРАВЛЕНИЯ ПРОЦЕССОМ ПРОИЗВОДСТВА ЭЛЕКТРОННЫХ АППАРАТОВ
}

Анотація. В результаті проведених досліджень, на підставі методу аналізу ієрархій, були зібрані статистичні дані за експертними оцінками параметрів, визначені управлінські витрати від впровадження модифікованого технологічного процесу. Проаналізовані складові управлінських витрат, обумовлені необхідністю залучення експертів, опитування великої кількості підприємств для збору статистики і узгодження виданих системою рекомендацій 3 передбачуваним ТП виробництва. Визначений функціонал ефективності впровадження модифікації в існуючий процес виробництва, досліджена динаміка даного функціоналу для різних за призначенням, умовам експлуатації і конструктивному оформленню технологічних об'єктів.

Ключові слова: спосіб управління, процес виробництва, електронний апарат, система підтримки прийняття рішень, продуктивність процесу виробництва, моделі автоматизованого керування.

\section{Введение}

Исследуя проблему организации автоматизированного управления технологическими процессами (ТП) производства сложноорганизованных объектов, следует большое внимание уделить вопросам обеспечения адаптации рассматриваемых процессов к особенностям производимого объекта, возможной ориентации технологического процесса на назначение и компоновку производимого изделия [1]. Решение данных вопросов следует искать в организации автоматизированного управления на основе интеллектуальных систем, но вначале необходимо отследить и обосновать необходимость применения данных подходов, проанализировать их экономическую целесообразность и оценить организационную необходимость подобного рода новшеств. С этой целью, для системного анализа вносимых в существующий технологический процесс новшеств, выбирают один из методов системного анализа, например метод анализа иерархий [2, 3]. Предполагаемая надстройка возлагает на себя: выполнение функций моделирования посредством программно-технических комплексов, работу экспертной системы на основании правил продукции, согласование выдаваемых экспертной системой рекомендаций с существующими нормами и правилами, действующими на предприятии, постпроцессирование управляющих воздействий для реализации принятых решений в ТП. Данная схема работает, как для ТП производства электронных аппаратов

(C) В.М. Смолій 
Міжвідомчий науково-технічний збірник «Адаптивні системи автоматичного управління» № 2' (33) 2018

(ЭА), так и для ТП производства любого сложноорганизованного объекта, например, плиты перекрытия промышленного здания с оптимизацией длины арматурной структуры при обеспечении заданной прочности, поэтому тематика проводимых работ является актуальной.

Целью исследований является решение актуальной научно-технической проблемы генерации адаптивного управления технологическим процессом производства электронных аппаратов, опирающегося на систему поддержки принятия решения решающую вопросы моделирования, анализа и генерации рекомендаций, применение которой позволяет повысить качество и надежность ЭА при оптимизации технико-экономических показателей всего процесса производства в целом.

\section{Основное содержание и результаты работы}

Рассматривая задачу управления процессом производства электронных аппаратов, следует выделить в ее рассмотрении следующие аспекты: необходимость включения в технологический процесс предлагаемой «надстройки» для моделирования свойств и параметров ЭА [1], составление по результатам моделирования некоторых заключений о приемлемости компоновки и параметров изделия и в противном случае необходимость генерации сценариев выхода из нежелательных ситуаций, организация управления процессом производства с учетом выдаваемых рекомендаций [4].

Таблицุа 1. Шкала интенсивности МАИ

\begin{tabular}{|c|l|}
\hline Величина & \multicolumn{1}{|c|}{ Значение } \\
\hline 1 & Равная важность \\
\hline 2 & $\begin{array}{l}\text { Промежуточное значение между равной важностью } \\
\text { и умеренным превосходством }\end{array}$ \\
\hline 3 & Умеренное превосходство одного над другим \\
\hline 4 & $\begin{array}{l}\text { Промежуточное значение между умеренным } \\
\text { и существенным превосходством }\end{array}$ \\
\hline 5 & Существенное превосходство одного над другим \\
\hline 6 & $\begin{array}{l}\text { Промежуточное значение между существенным } \\
\text { и значительным превосходством }\end{array}$ \\
\hline 7 & Значительное превосходство одного над другим \\
\hline 8 & $\begin{array}{l}\text { Промежуточное значение между значительным } \\
\text { и очень сильным превосходством }\end{array}$ \\
\hline 9 & Очень сильное превосходство одного над другим \\
\hline
\end{tabular}

После иерархического воспроизведения проблемы [4] устанавливаются приоритеты критериев и оценивается каждая из альтернатив по критериям. В МАИ элементы задачи сравниваются попарно по отношению к их воздействию на общую для них характеристику. Система парных сведений приводит 
к результату, который может быть представлен в виде обратно симметричной матрицы. Элементом матрицы а(i,j) является интенсивность проявления элемента иерархии і относительно элемента иерархии j, оцениваемая по шкале интенсивности от 1 до 9, где оценки имеют смысл, приведенный в табл. 1.

Если при сравнении одного фактора i с другим j получено $a(\mathrm{i}, \mathrm{j})=\mathrm{b}$, то при сравнении второго фактора с первым получаем $\mathrm{a}(\mathrm{j}, \mathrm{i})=1 / \mathrm{b}$. Относительная сила, величина или вероятность каждого отдельного объекта в иерархии определяется оценкой соответствующего ему элемента собственного вектора матрицы приоритетов, нормализованного к единице. Процедура определения собственных векторов матриц поддается приближению с помощью вычисления геометрической средней.

Приоритеты синтезируются начиная со второго уровня вниз. Локальные приоритеты перемножаются на приоритет соответствующего критерия на вышестоящем уровне и суммируются по каждому элементу в соответствии с критериями, на которые воздействует элемент.

Важным понятием теории является так называемый индекс согласованности (ИС), который дает информацию о степени нарушения согласованности

$$
И C=\left(\lambda_{\max }-n\right) /(n-1),
$$

где $n$ - размерность матрицы, а $\lambda_{\max }$ считается следующим образом: вначале суммируется каждый столбец суждений, затем сумма первого столбца умножается на величину первой компоненты нормализованного вектора приоритетов, сумма второго столбца - на вторую компоненту и т.д., затем полученные числа суммируются. $\lambda_{\max } \geq \mathrm{n}$.

Теперь необходимо сравнить ИС с той величиной, которая получилась бы при случайном выборе суждений. Значения этой величины - случайной согласованности (СС) табулированы.

Определяя ИС и СС, находим отношение согласованности

$$
O C=\frac{И C}{C C} .
$$

Если для конкретной матрицы окажется, что ОС >0.1, то можно утверждать, что суждения эксперта, на основе которых заполнена исследуемая матрица, сильно рассогласованы, и необходимо либо сменить экспертов, либо найти дополнительные данные, либо решать проблему другим методом.

Матрица парных сравнений для уровня иерархии исследования управленческих издержек, обусловленных необходимостью привлечения экспертов для создания специализированных баз знаний и правил продукции имеет вид, приведенный в табл. 2. В табл.2 и последующих введены следующие обозначения: 
Міжвідомчий науково-технічний збірник «Адаптивні системи автоматичного управління» № 2' (33) 2018 О1 - бытовой ЭА, О2 - стационарный ЭА, О3 - транспортный ЭА, О4 - военный ЭА, О5 - авиационный ЭА, О6 - космический ЭА.

Таблица 2. Матрица парных сравнений для исследования управленческих издержек

\begin{tabular}{|c|c|c|c|c|c|c|c|c|}
\hline $\begin{array}{c}\text { Необходимость } \\
\text { привлечения экспертов }\end{array}$ & O1 & O2 & O3 & O4 & O5 & O6 & $\begin{array}{c}\text { Собственный } \\
\text { вектор }\end{array}$ & $\begin{array}{c}\text { Вектор } \\
\text { приоритетов }\end{array}$ \\
\hline O1 & 1 & $1 / 3$ & $1 / 5$ & $1 / 7$ & $1 / 9$ & $1 / 8$ & 0,226 & 0,025 \\
\hline O2 & 3 & 1 & $1 / 2$ & $1 / 4$ & $1 / 5$ & $1 / 7$ & 0,47 & 0,052 \\
\hline O3 & 5 & 2 & 1 & $1 / 3$ & $1 / 6$ & $1 / 5$ & 0,693 & 0,076 \\
\hline O4 & 7 & 4 & 3 & 1 & $1 / 4$ & $1 / 3$ & 1,383 & 0,152 \\
\hline O5 & 9 & 5 & 6 & 4 & 1 & $1 / 2$ & 2,854 & 0,315 \\
\hline O6 & 8 & 7 & 5 & 3 & 2 & 1 & 3,448 & 0,38 \\
\hline
\end{tabular}

Для рассматриваемого случая максимальное собственное значение матрицы $\lambda \max =6,398$ и для $\mathrm{n}=6$ величина случайной согласованности составляет 1,24 , поэтому отношение согласованности для рассматриваемой матрицы равно 0,064, что меньше допустимого $(0,08 \div 0,1)$, поэтому пересмотр суждений не требуется. Анализируя заполненную экспертами табл. 2 получаем, что максимальный вклад в управленческие издержки за счет необходимости привлечения экспертов имеет космический ЭА так как процесс его компоновки, подбора материалов и сборки основан на привлечении высококвалифицированных специалистов и формализация этих процедур требует привлечения экспертов с не меньшей квалификацией и опытом работы. Наименьшую составляющую в управленческих издержках за счет привлечения экспертов имеют бытовые ЭА т.к. для их производства характерен выигрыш от накопления информации в базах данных по выполненным проектам и отсутствуют наукоемкие производственные процессы.

В табл. 3. сведены статистически обработанные знания экспертов по оценкам параметров для уровня иерархии исследования управленческих издержек, получаемых за счет необходимости задействования большого количества предприятий для сбора статистики по формализации принимаемых решений, тестировании уже принятых и построению правил продукции для системы автоматизированного управления производством ЭА.

Максимальное собственное значение матрицы $\lambda \max =6,518$ и для $n=6$ величина случайной согласованности составляет 1,24, поэтому отношение согласованности для рассматриваемой матрицы равно 0,083, что меньше допустимого, поэтому пересмотр суждений не требуется. Исследуя закономерности табл. 2 получаем, что наибольшие управленческие издержки за счет необходимости задействования большого количества предприятий для сбора статистики имеют ЭА космического назначения т.к. собираемые статистические данные касаются, 
как космических аппаратов различного назначения, так и их различных серий и сложившихся технологий производства. Наименьшие издержки характерны для бытовых ЭА, что обусловлено относительной простотой принимаемых конструкторско-технологических решений и их сравнительной тождественностью, тиражируемой от предприятия к предприятию.

Таблища 3. Матрица парных сравнений для исследования управленческих издержек

\begin{tabular}{|l|l|l|l|l|l|l|l|l|}
\hline $\begin{array}{c}\text { Необходимость } \\
\text { задействовать большое ко- } \\
\text { личество предприятий } \\
\text { для сбора статистики }\end{array}$ & $\mathbf{O 1}$ & $\mathbf{O 2}$ & $\mathbf{O 3}$ & $\mathbf{O 4}$ & $\mathbf{O 5}$ & $\mathbf{O 6}$ & $\begin{array}{c}\text { Собст- } \\
\text { венный } \\
\text { вектор }\end{array}$ & $\begin{array}{c}\text { Вектор } \\
\text { приори- } \\
\text { тетов }\end{array}$ \\
\hline $\mathrm{O} 1$ & 1 & 1 & $1 / 3$ & $1 / 4$ & $1 / 6$ & $1 / 5$ & 0,375 & 0,048 \\
\hline $\mathrm{O} 2$ & 1 & 1 & $1 / 2$ & $1 / 3$ & $1 / 5$ & $1 / 4$ & 0,45 & 0,057 \\
\hline $\mathrm{O} 3$ & 3 & 2 & 1 & $1 / 2$ & $1 / 3$ & $1 / 4$ & 0,794 & 0,101 \\
\hline $\mathrm{O} 4$ & 4 & 3 & 2 & 1 & $1 / 4$ & $1 / 2$ & 1,201 & 0,153 \\
\hline $\mathrm{O} 5$ & 6 & 5 & 3 & 4 & 1 & $1 / 3$ & 2,221 & 0,283 \\
\hline $\mathrm{O} 6$ & 5 & 4 & 4 & 2 & 3 & 1 & 2,798 & 0,357 \\
\hline
\end{tabular}

Сравнительный анализ управленческих издержек, обусловленных необходимостью согласования выдаваемых системой рекомендаций с предполагаемым ТП производства, приведен в табл. 4.

Таблица 4. Матрица парных сравнений для исследования управленческих издержек

\begin{tabular}{|l|l|l|l|l|l|l|l|l|}
\hline $\begin{array}{c}\text { Необходимость согласования } \\
\text { выдаваемых системой } \\
\text { рекомендаций с предполагаемым } \\
\text { ТП производства }\end{array}$ & $\mathbf{O 1}$ & $\mathbf{O 2}$ & $\mathbf{O 3}$ & $\mathbf{O 4}$ & $\mathbf{O 5}$ & $\mathbf{O 6}$ & $\begin{array}{c}\text { Собст- } \\
\text { венный } \\
\text { вектор }\end{array}$ & $\begin{array}{c}\text { Вектор } \\
\text { приори- } \\
\text { тетов }\end{array}$ \\
\hline O1 & 1 & $1 / 5$ & $1 / 6$ & $1 / 7$ & $1 / 8$ & $1 / 9$ & 0,201 & 0,021 \\
\hline O2 & 5 & 1 & $1 / 4$ & $1 / 6$ & $1 / 7$ & $1 / 8$ & 0,394 & 0,041 \\
\hline O3 & 6 & 4 & 1 & $1 / 4$ & $1 / 5$ & $1 / 6$ & 0,765 & 0,08 \\
\hline O4 & 7 & 6 & 4 & 1 & $1 / 3$ & $1 / 4$ & 1,552 & 0,163 \\
\hline O5 & 8 & 7 & 5 & 3 & 1 & $1 / 2$ & 2,737 & 0,287 \\
\hline O6 & 9 & 8 & 6 & 4 & 2 & 1 & 3,888 & 0,408 \\
\hline
\end{tabular}

Для приведенных в табл. 4 данных получаем, что максимальное собственное значение матрицы $\lambda \max =6,519$, величина случайной согласованности составляет 1,24 (n=6), отношение согласованности равно 0,084, что меньше допустимого, поэтому пересмотр суждений не требуется. Анализируя закономерности, сведенные в табл. 4, получаем, что наибольший вес управленческих издержек связанных с необходимостью согласования выдаваемых системой рекомендаций с предполагаемым ТП производства имеют ЭА космического назначения, а наи- 
Міжвідомчий науково-технічний збірник «Адаптивні системи автоматичного управління» № 2' (33) 2018

меньший - бытовые ЭА. Подобного рода тенденция для первых обусловлена необходимостью адаптации сценариев работы экспертной системы под установившиеся традиции и навыки специалистов-производственников. Небольшой приоритет рассматриваемого фактора для бытовых и стационарных ЭА обусловлен легкой формализацией процесса принятия решений и относительной простотой принимаемых решений.

Функционал эффективности внедрения модификации в существующий процесс производства [4] складывается из

$$
J=J_{1}+J_{2}
$$

где $J_{1}, J_{2}$ - составляющие функционала, обусловленные выгодами и издержками от внедрения предлагаемых ПТК для моделирования механических нагрузок ЭА и их составляющих (модифицированный технологический процесс производства).

Составляющие функционала эффективности [4], определяемые на основании метода анализа иерархий $[2,3]$, имеют вид

$$
\begin{gathered}
J_{1}=0.167 \cdot Э B+0.833 \cdot У B, \\
J_{2}=0.75 \cdot Э И+0.25 \cdot У И,
\end{gathered}
$$

где ЭВ, УВ - экономические и управленческие выгоды, получаемые от внедрения предлагаемых модификаций в существующий технологический процесс производства ЭА, ЭИ, УИ - соответственно экономические и управленческие издержки, получаемые от внедрения предлагаемых модификаций в существующий технологический процесс производства ЭА.

Зависимости показателей функционала эффективности от признаков, проанализированных методом анализа иерархий, имеют вид

$$
\left\{\begin{array}{l}
Э В=0.512 \cdot \Pi p 1+0.135 \cdot \Pi p 2+0.307 \cdot \Pi p 3+0.047 \cdot \Pi p 4 \\
У B=0.102 \cdot \Pi p 5+0.172 \cdot \Pi p 6+0.726 \cdot \Pi p 7 \\
Э И=0.428 \cdot \Pi p 8+0.227 \cdot \Pi p 9+0.176 \cdot \Pi p 10+0.12 \cdot \Pi p 11+0.049 \cdot \Pi p 12 \\
У И=0.111 \cdot \Pi p 13+0.22 \cdot \Pi p 14+0.667 \cdot \Pi p 15
\end{array}\right.
$$

где Пр1 - Пр15 - непосредственно анализируемые акторы для исследования рассматриваемой иерархии проблемы.

С учетом результатов обработки статистических выборок, полученных после опроса экспертов ( табл. 2 - табл. 4), получаем

$$
\left\{\begin{array}{l}
\Pi p 13=0.025 \cdot O 1+0.052 \cdot O 2+0.076 \cdot O 3+0.152 \cdot O 4+0.315 \cdot O 5+0.38 \cdot O 6 \\
\Pi p 14=0.048 \cdot O 1+0.057 \cdot O 2+0.101 \cdot O 3+0.153 \cdot O 4+0.283 \cdot O 5+0.357 \cdot O 6 \\
\Pi p 15=0.021 \cdot O 1+0.041 \cdot O 2+0.08 \cdot O 3+0.163 \cdot O 4+0.287 \cdot O 5+0.408 \cdot O 6
\end{array}\right.
$$

где О1 - О6 - непосредственно объекты исследования, манипулируя параметрами и компоновкой которых, влияют на признаки и, наоборот, рассматривая и исследуя политику акторов в отношении объектов влияют на политику и фокус рассматриваемой проблемы. 
Систематизировав все имеющиеся сведения об объекте исследований, рассматривая статистически обработанные экспертные оценки экономических и управленческих выгод и издержек, получают следующую динамику анализируемых признаков исследуемых типов ЭА, приведенную в табл. 5.

Таблица 5. Экономические и управленческие признаки для ЭА различного назначения

\begin{tabular}{|c|c|c|c|c|c|c|}
\hline $\begin{array}{l}\text { Тип ЭА } \\
\text { Признак }\end{array}$ & 01 & $\mathbf{O 2}$ & $\mathbf{O 3}$ & 04 & 05 & O6 \\
\hline$\Pi p 1$ & 0.469 & 0.247 & 0.12 & 0.051 & 0.079 & 0.034 \\
\hline Пр2 & 0.436 & 0.223 & 0.147 & 0.087 & 0.045 & 0.062 \\
\hline Пр3 & 0.04 & 0.046 & 0.112 & 0.153 & 0.285 & 0.364 \\
\hline Пр4 & 0.029 & 0.047 & 0.072 & 0.163 & 0.276 & 0.412 \\
\hline Пр5 & 0.029 & 0.048 & 0.084 & 0.124 & 0.257 & 0.457 \\
\hline Пр6 & 0.038 & 0.054 & 0.086 & 0.157 & 0.286 & 0.378 \\
\hline Пр7 & 0.04 & 0.054 & 0.088 & 0.165 & 0.222 & 0.432 \\
\hline Пр8 & 0.417 & 0.272 & 0.141 & 0.089 & 0.058 & 0.024 \\
\hline Пр9 & 0.37 & 0.275 & 0.17 & 0.087 & 0.056 & 0.043 \\
\hline Пр10 & 0.368 & 0.292 & 0.143 & 0.081 & 0.081 & 0.34 \\
\hline Пр11 & 0.03 & 0.056 & 0.097 & 0.161 & 0.216 & 0.44 \\
\hline Пр12 & 0.459 & 0.255 & 0.102 & 0.076 & 0.072 & 0.035 \\
\hline Пр13 & 0.025 & 0.052 & 0.076 & 0.152 & 0.315 & 0.38 \\
\hline Пр14 & 0.048 & 0.057 & 0.101 & 0.153 & 0.283 & 0.357 \\
\hline Пр15 & 0.021 & 0.041 & 0.08 & 0.163 & 0.287 & 0.408 \\
\hline
\end{tabular}

Величины промежуточных функционалов выгод и издержек, а также функционала эффективности внедрения модификации в существующий процесс производства (3) проиллюстрированы в табл. 6.

Анализируя результаты исследования функционала эффективности внедрения модификации в существующий процесс производства, получают, что такое новшество наиболее эффективно для ЭА космического и бытового назначения, а наименее эффективно для технологического процесса производства транспортных ЭА. Данные тенденции объясняются тем, что у космических ЭА большая часть производственной информации систематизируется вводимыми новшествами, формализуются имеющиеся знания и технические наработки существующего высокотехнологического процесса производства, и испытания опытного образца изделия в предполагаемых условиях эксплуатации осуществляются на модели, без циклов возврата производимого изделия на доработку. Для бытового ЭА также характерны подобные факторы достижения управленческих и экономических выгод от предполагаемого внедрения. 
Таблица 6. Функционал эффективности внедрения модификации в существующий процесс производства

\begin{tabular}{|c|l|l|l|l|l|l|l|}
\hline Тип ЭА \\
Показатель & ЭВ & УВ & ЭИ & УИ & $\mathbf{J}_{\mathbf{1}}$ & $\mathbf{J}_{\mathbf{2}}$ & Ј \\
\hline O1 & 0.313 & 0.039 & 0.353 & 0.027 & 0.084 & 0.272 & 0.178 \\
\hline O2 & 0.173 & 0.053 & 0.249 & 0.046 & 0.073 & 0.199 & 0.136 \\
\hline O3 & 0.119 & 0.087 & 0.141 & 0.084 & 0.093 & 0.127 & 0.11 \\
\hline O4 & 0.092 & 0.159 & 0.095 & 0.159 & 0.148 & 0.111 & 0.13 \\
\hline O5 & 0.147 & 0.237 & 0.081 & 0.289 & 0.222 & 0.133 & 0.177 \\
\hline O6 & 0.157 & 0.425 & 0.134 & 0.393 & 0.38 & 0.199 & 0.29 \\
\hline
\end{tabular}

\section{Заключение}

В результате проведенных исследований, на основании метода анализа иерархий, были собраны статистические данные по экспертным оценкам параметров, определены управленческие издержки от внедрения модифицированного технологического процесса, проанализированы факторы необходимости привлечения экспертов, опроса большого количества предприятий (и экспертов на предприятии) для сбора статистики, оценена необходимость согласования выдаваемых системой рекомендаций с предполагаемым ТП производства. Определен функционал эффективности внедрения модификации в существующий процесс производства. Наибольший эффект от внедрения предлагаемого модифицированного технологического процесса будут иметь ЭА космического и бытового назначения, наименьший - ЭА транспортного назначения, т.е. данную систему необходимо развивать и в дальнейшем применить математический аппарат оптимизации свойств и параметров изделия для достижения оптимального значения экономико-управленческого функционала (3), что позволит управлять процессом производства с минимальными затратами при максимальном управленческом эффекте, а также разработать механизм влияния этих параметров на структуру и параметры технологического объекта.

\section{Список використаних джерел}

1. Смолий В.Н. Управление производством сложноорганизованных технологических объектов//Вісник СНУ ім.В.Даля.- 2009. - № 2 (132). Ч.2. - С. 46 - 55.

2. Смолий В.Н. Исследование эффективности управления процесса производства электронных аппаратов // Прогресивні технології і системи машинобудування: Міжнародний зб. наукових праць. - Донецьк: ДонНТУ, 2010.- Вип. 39. - С. 174 - 178.

3. Смолий В.Н. Особенности концепции управления производством электронных аппаратов// Вісник СНУ ім.В.Даля. - 2010. - № 2 (144). - С. 128 - 133.

4. Vitaly Ulshin, Victoria Smoliy. Automated management by designer preparation of production of electronic vehicles/ TEKA Kom. Mot. I Energ. Roln. - OL PAN, 2011, 11A, P.276- 281 .

5. Ульшин В.А., Смолий В.Н. Функции лица, принимающего решение, при управлении производством электронных аппаратов// Вісник СНУ ім.В.Даля. - 2011. № 3 (157) - c. 214 - 220 . 